\title{
Outreach in paediatric radiology: European activities
}

\author{
Rutger A. J. Nievelstein • Catherine M. Owens \\ On behalf of the officers of the European Society of \\ Paediatric Radiology (ESPR)
}

Received: 28 November 2013 / Accepted: 5 February 2014

(C) Springer-Verlag Berlin Heidelberg 2014

During the last decade, globalisation has resulted in an international focus on public health care, in particular the ongoing disparity between health care systems in resource-rich and resource-poor countries. Because radiology plays an important role in the diagnosis and treatment of disease, involvement of radiologists in regional health care programs is of crucial importance on an urgent basis. Many of the problems - and some solutions - related to health care improvement in developing countries are relevant to both adults and children; however imaging in children requires dedicated equipment, with specific safety considerations related to the use of ionising radiation, and specialised knowledge of paediatric diseases.

For many decades, European paediatric radiologists have been actively involved in worldwide outreach initiatives, on an individual basis and through international regional and global aid organisations. Their current involvement mainly focuses on on-site education, hands-on training and diagnostic consultation (more recently via teleradiology services). However, to facilitate effective and sustainable implementation of effective childfocussed radiology worldwide, collaboration amongst institutional, local, regional and international organisations is needed.

Through these collaborations, it will become possible to provide resource-poor regions with the basic equipment, the level of technological expertise and the educational resources needed to guarantee adequate diagnostic imaging of children. The educational resources required include on-site lectures and courses, on-site hands-on training, online learning systems and collaborative case reviews. In addition, this education should involve and engage all those involved in medical imaging: local and volunteer personnel including residents, radiologists, other physicians and technologists.

The foundation of the World Federation of Pediatric Imaging in 2011 (with ESPR as one of the founding societies) is a fundamentally important global initiative that "provides an international platform for pediatric radiology organizations united to address the challenges in global pediatric imaging, training and the delivery of services" [1]. Within the European Union, the European Society of Paediatric Radiology (ESPR) has had a well-established collaboration for many years with the European Society of Radiology via the European School of Radiology (ESOR) (www.esor.org). This particular collaboration focuses on organising both basic and advanced imaging courses in areas of perceived need in Eastern and Central European countries, as well as offering exchange programmes for paediatric imaging fellowships to centres of excellence within the European Union. Such collaborations are among our most important missions at ESPR.

Conflicts of interest None

\section{References}

1. World Federation of Pediatric Imaging (2012) Strategic framework. WFPI Web site. http://www.wfpiweb.org/Portals/7/About/WFPI Strategic\%20Framework\%20_Full_version.pdf. Accessed 22 Jan $201 \overline{4}$

\author{
R. A. J. Nievelstein $(\square)$ \\ Department of Pediatric Radiology, UMC Utrecht, \\ P.O. Box 85500, 3508 GA Utrecht, The Netherlands \\ e-mail: R.A.J.Nievelstein@umcutrecht.nl \\ C. M. Owens \\ Department of Radiology, \\ Great Ormond Street Hospital for Children NHS Trust, \\ London, UK
}

